\title{
Note sur la déclinaison de 74 Cygni.
}

1. Au mois d'octobre 1884 j'observai 8 étoiles zénithales dont 4 au Sud et 4 au Nord du zénith de Capodimonte et chacune d'elles en 7 positions du cercle divisé en faisant pour chaque étoile 14 observations dont la moitié faisant face au Nord et lautre moitié faisant face au Sud. Comme les étoiles observées sont toutes voisines au zénith, une erreur qui dérive de la position de l'observateur, est presque totalement éliminée dans la moyenne des deux observations conjuguées faites dans la même position du cercle divisé. Je donne ici les distances zénithales réduites à l'époque intermédiaire des observations, c'est-a-dire, au 19 octobre $\mathbf{1 8 8 4}$, et en regard, les déclinaisons apparentes pour la même époque, corrigées des petits termes lunaires. Les déclinaisons sont extraites du Berliner Jahrbuch.

\begin{tabular}{|c|c|c|c|c|c|c|c|}
\hline & & & \multicolumn{3}{|c|}{$\delta$} \\
\hline$\nu$ & Cygni & $+0^{\circ}$ & & 55.04 & $+40^{\circ}$ & $43^{\prime}$ & 51.2 \\
\hline$\xi$ & Cygni & -2 & $3^{6}$ & 46.08 & +43 & 21 & 31.57 \\
\hline 74 & Cygni & +0 & 57 & 35.66 & +39 & 54 & .05 \\
\hline$\pi$ & Cygni & -7 & 55 & I $4.4 \mathrm{I}$ & +48 & 46 & 59.71 \\
\hline$\pi$ & Pegasi & +8 & 14 & $3^{8 \cdot 39}$ & +32 & 37 & 7.9 \\
\hline Io & Lacertae & +2 & 24 & $23.7 \mathrm{I}$ & +38 & 27 & 22.22 \\
\hline 13 & Lacertae & -0 & $2 \mathrm{I}$ & 26.67 & $+4 I$ & I 3 & 12.15 \\
\hline 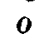 & Andromedae & -0 & 50 & $57 \cdot 5^{6}$ & $+4 I$ & 42 & 42.8 \\
\hline
\end{tabular}

Or, si sans admettre aucune valeur pour la latitude instrumentale on construit les $\Delta \delta$ et les $\Delta \zeta$ pour les étoiles méridionales et pour les septentrionales entre elles, on a pour $(\Delta \delta-\Delta \zeta)$ :

$$
\begin{aligned}
& v \text { Cygni - 74 Cygni. } \quad+1.60 \\
& \nu \text { Cygni }-\pi \text { Pegasi }-0.02 \\
& v \text { Cygni - Io Lacertae } \quad+0.38 \\
& 74 \text { Cygni }-\pi \text { Pegasi }-1.62 \\
& 74 \text { Cygni - 10 Lacertae - } 1.22 \\
& \text { ro Lacertae }-\pi \text { Pegasi } \quad-0.40 \\
& \pi^{2} \text { Cygni - } \xi \text { Cygni } \quad-0.19 \\
& \pi^{2} \text { Cygni - } 0 \text { Andromedae }-0.02 \\
& \pi^{2} \text { Cygni -1 } 3 \text { Lacertae }-0.18 \\
& \xi \text { Cygni - a Andromedae +0.1 } 7 \\
& \xi \text { Cygni - } 13 \text { Lacertae +o.01 } \\
& \text { o Andromedae - I3 Lacertae - } 36
\end{aligned}
$$

On voit dans ce tableau que seulement où se trouve l'étoile 74 Cygni, il y a des différences inconciliables avec la qualité des observations et les résultats des autres étoiles. Si l'on faisait à la déclinaison de 74 Cygni la correction $+\mathrm{I}: 20$, les différences qui regardent l'étoile deviendraient:

$$
\begin{array}{clll}
v & \text { Cygni }-74 & \text { Cygni } & +0.40 \\
74 & \text { Cygni }-\pi \text { Pegasi } & -0.42 \\
74 \text { Cygni }-10 \text { Lacertae } & -0.02
\end{array}
$$

C'est-à-dire que des observations de 1884 on tire un soupçon très fondé que cette déclinaison soit en erreur de - I" au moins.

2. En septembre 1885 j'ai observé l'étoile avec les trois autres I 5 Cygni, $v$ Cygni, Io Lacertae qui culminent aussi un peu au Sud du zénith de Capodimonte et qui la comprennent en $\alpha$ et $\delta$. Les distances zénithales, réduites au 16 septembre (chacune moyenne de 7 observations en 7 positions du cercle) donnent le tableau:

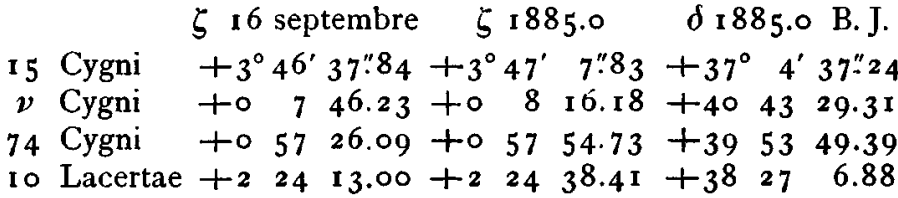
d'où l'on tire:

$$
\begin{aligned}
& \Delta \delta \quad \Delta \zeta \\
& 74 \text { Cygni- I } 5 \text { Cygni +2 }{ }^{\circ} 49^{\prime} \text { I 2". } 15-2^{\circ} 49^{\prime} \text { I 3". } 10-0.95 \\
& 74 \text { Cygni- } v \text { Cygni -o } 4939.92 \text { to } 4938.55 \text { - } 1.37 \\
& 74 \text { Cygni-10 Lacertae +I } 2642.51-12643.68 \text { - I. } 17 \\
& \text { Moyenne }=-\mathbf{I} . \mathbf{1} 6 \text {. }
\end{aligned}
$$

Donc, selon ces observations et celles de 1884 , la déclinaison de 74 Cygni*) dans le système du Berliner Jahrbuch devrait être corrigée d'environ +1". 2 .

Dans un mémoire qui est sous presse, on verra que la latitude qui résulte de 74 Cygni non corrigée est inconciliable avec celle que l'on tire des autres étoiles.

$$
\text { Cåpodimonte } 1885 \text { Oct. } 2 \text {. }
$$

\section{A. Nobile.}

*) Die Position dieses Sterns in Auwers' Fund. Cat. der A. G., auf den sich das System des Rerl. Jahrb. gründet, beruht nur auf dem Manuscript-Catalog Pulkowa 1871 und 3 Greenwicher Beobachtungen, und muss daher zu den weniger sicheren des Catalogs gerechnet werden. Kr.

\section{Planet (252).}

Nach einer Mittheilung des Herrn Perrotin in den Comptes Rendus 1885 Oct. 26 ist dieser Planet, von welchem in Nr. 2690 eine telegraphisch übermittelte Beobachtung vom 27. October mitgetheilt wird, bereits am r1. Oct. in Nizza entdeckt, aber zunächst für den Planeten (2 23) Rosa gehalten worden.

Die beiden ersten Beobachtungen des Herrn Perrotin lauten:

$$
\begin{aligned}
& 1885 \text { Oct. I } 11_{1}^{\mathrm{h}} 5^{6^{\mathrm{m}} 46^{\mathrm{s}}} \text { M. Z. Nizza } \alpha \text { app. }=1^{\mathrm{h}} \mathrm{I}^{\mathrm{m}} 30^{\mathrm{s}} .7 \quad \delta \text { app. }=+9^{\circ} \quad 6^{\prime} 57^{\prime \prime} \circ
\end{aligned}
$$

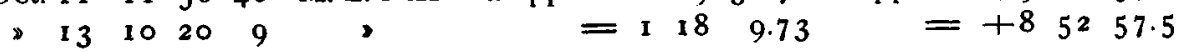

Die Position vom 11. Oct. ist nur eine genäherte. Der Planet ist 13. Grösse. 